\title{
Le labyrinthe et les nuées : les espaces aberrants de Kurosawa
}

\section{Serge Chauvin}

\section{(2) OpenEdition}

\section{Journals}

\section{Édition électronique}

URL : http://journals.openedition.org/shakespeare/183

DOI : $10.4000 /$ shakespeare.183

ISSN : 2271-6424

Éditeur

Société Française Shakespeare

\section{Édition imprimée}

Date de publication : 1 novembre 1998

Pagination : 31-35

ISBN : 2-84269-230-6

\section{Référence électronique}

Serge Chauvin, "Le labyrinthe et les nuées : les espaces aberrants de Kurosawa », Actes des congrès de la Société française Shakespeare [En ligne], 16 | 1998, mis en ligne le 01 novembre 2007, consulté le 01 mai 2019. URL : http://journals.openedition.org/shakespeare/183 ; DOI : 10.4000/shakespeare.183 


\section{S H A K E S P E A R E \\ \& $\quad$ L E $\quad$ C I N É M A}

Société Française Shakespeare

Actes du Congrès de 1998

米 $*$ *

Textes réunis et présentés par

Patricia DORVAL

publiés sous la direction de

Jean-Marie MAGUIN 
Site web : <http : //alor.univ-montp3.fr/serinf/SFS/> Liste de diffusion : <sfs-1@smrl.univ-montp3.fr >

Tous droits de traduction, de reproduction et d'adaptation réservés pour tous les pays.

(C) 1998. Société Française Shakespeare,

École Normale Supérieure, 45 rue d'Ulm. 75005 Paris.

ISBN 2-84269-230-6 


\section{LE LA B Y R IN THE ET LES NUÉES: L E S E S PACES A B ER RA N T S DE K U R O S A W A}

Les deux adaptations shakespeariennes de Kurosawa, Le château de l'araignée et Ran, inspirées respectivement de Macbeth et de King Lear, ne se contentent pas de transposer l'intrigue des pièces dans le Japon féodal, mais se singularisent par une liberté extrême à l'égard du matériau d'origine $(R a n$ attribuant au vieux roi des fils plutôt que des filles) et surtout du texte de théâtre. De Macbeth, Le château de l'araignée, qui seul nous occupe ici, ne conserve que la trame, prétexte à une rêverie plastique qui en fait l'un des films les moins loquaces jamais suscités par Shakespeare. De fait, cette éclipse du verbe rend ici quelque peu inopérant le vieux débat post-bazinien sur la théâtralité à l'écran, selon lequel le cinéma trouverait son essence même en respectant la convention scénique d'un espace unique et de pure convention (loin de toute velléité d' «aérer» la pièce, ou de la "naturaliser») et d'une continuité temporelle, se présentant comme le lieu d'une profération du texte. Kurosawa, lui, construit, invente un espace, indissociablement scénographique et topographique, qui n'est concevable qu'en fonction des possibilités du cinéma, et qui pourtant rejoint, in extremis, la vérité du théâtre. C'est ce qu'on s'efforcera de montrer par une approche empirique sinon linéaire, et circonscrite au film même, laissant de côté l'influence sur Kurosawa d'une tradition japonaise aussi bien théâtrale (le nô) que picturale, que notre méconnaissance sans faille du sujet nous interdit d'étudier ici.

La perte de tous repères, l'égarement spatial autant que moral ou mental sont évidemment inhérents à la problématique de Macbeth, et nourrissaient déjà l'adaptation d'Orson Welles. La succession des espaces, leur substitution 
mutuclle offrent un équivalent à la scène comme lieu constamment redéfini ; la caméra ne sert pas à homogénéiser l'espace, ni à le rendre fluide. Chez Kurosawa, le film convoque son espace propre, l'invoque au sens du médium : un espace aussi éloigné de l'artifice revendiqué de la scène que du référent concret du film (le château montré à l'écran, dans sa matérialité de décor). On assiste ici à un double phénomène : la restitution de l'espace scénique par la stylisation et le dépouillement (par «soustraction», pourrait-on dire), où le lieu est suscité par la parole, et l'apparition d'un espace labile, fantastique, où s'opère le passage d'un monde à l'autre, dans une temporalité différente, et où se mêlent les morts et les vivants comme autant de «walking shadows». Ces deux opérations naissent d'un même principe de mise en scène : un jeu sur le champ-contrechamp qui commence par substituer à la frontalité scénique un modèle circulaire, puis qui, miné par l'ubiquité, aboutit à unc inversion où l'action est représentée du point de vue du surnaturel.

Le film s'ouvre sur un paysage dans le brouillard: est-ce la nuée originclle? L'hypothèse est aussitôt démentie par un chœur masculin off : cette vision désoléc est celle d'un monde d'après la catastrophe, d'un sol à jamais stérile, où scule perdure la vanité de toutes choses, l'échec de toute ambition, l'éternel retour d'un mal chaque fois à vaincre. Ce lieu n'offre de prime abord aucun repère, aucune trace humaine, aucun ancrage naturel même : les panoramiques interrompus par le montage, l'usage d'un fonducnchaîné comme émanant dirait-on des nuées, rendent cet espace inassignable. Lorsqu'enfin les brumes se dissipent, elles ne révèlent que les traces d'une absence : un monument funćraire, de rares vestiges de remparts réduits à leurs fondations. Ici une histoire a eu lieu, mais il n'en reste plus rien. Dans ces ruines, on n'a comme repère que la trace de son propre égarement. D'un nouvcau fondu-enchaîné, que la nuée rend presque imperceptible, vont alors se dessiner les vagues contours du château. Comme si la parole du chœur avait su créer l'illusion, guidant et relayant l'imagination du spectateur. Procédé cher au théâtre, si ce n'est que la scène est un espace vierge à peupler, où se recréc chaque soir un monde. Le château de l'araignée, lui, ne sera jamais qu'un théâtre de fantômes. La temporalité substitue donc à la prophétie l'invocation du passé, sa recréation (à l'inverse du Macbeth d'Orson Welles, dont les images de limon représentaient une création mythique du monde). Et les ossements qui jonchent la clairière où tisse la sorcière apparaissent comme une sorte de prophétie au futur antérieur.

Le lieu paradoxal, l'espace mouvant, c'est bien sûr au premier chef la forêt, théâtre privilégié de la dépossession de soi, de l'exposition aux puissances du monde, où l'on se perd pour se retrouver transformé. La chevauchée de Washizu/Macbeth et de Miki/Banquo dans la forêt marque le passage d'un monde à l'autre; la vitesse du galop, que les panoramiques ne suivent qu'au risque de perdre le point, les branchages qui entravent la vision, 
les raccords dans le mouvement, les éclairs de l'orage qui amènent et masquent tout à la fois les sautes d'un plan à l'autre : tout concourt à donner le sentiment que, malgré tout l'élan des cavaliers, la voie droite est perdue. Lors de cette première chevauchée, Washizu et Miki ne savent donc pas vraiment où ils vont, ce qui ne les empêche pas de foncer tête baisséc, d'aller «de l'avant» : non qu'ils progressent dans l'espace, mais ils sont guidés, voire aspirés, par une série de paliers, vers le monde des esprits. La forêt de toute façon est ce licu où la logique spatiale n'a plus cours : "La forêt est un labyrinthe. N'y suivez aucun sentier. Évitez-les. Foncez droit devant vous. Évitez les pistes». El de même que le chœur du prologue permettait par l'invocation le glissement du présent au passé, de même c'est le chant fredonné de la tisseuse qui par la prophétie fait apparaître le futur dans le présent, et basculer le réel dans la magie. La voix de la tisseuse ne paraît pas s'originer dans son corps : elle flotte, comme détachée de lui, et semble émaner de la forêt, en une fuyante ubiquité. Décalage renforcé naturellement par le choix, conforme à la tradition théâtrale japonaise, d'un acteur travesti pour interpréter la sorcière. Cette créature que seul atteste le poids de sa parole évolue donc entre deux sexes comme entre deux mondes, impossible somme d'une voix fuyante et d'un corps volatil.

La clairière, si inattendue soit-elle, est d'abord pourtant un espace doté de profondeur, où l'on peut s'approcher de la hutte de la tisseuse. Puis ce mouvement s'immobilise et cède le pas à une frontalité scénique. Mais le jeu du champ-contrechamp, d'abord neutre en apparence (pur enchaînement causal permettant de rendre visible la réaction des deux hommes à la prophétic) bascule lorsque les guerriers sont filmés d'un point de vue inassignable : un regard qui s'originerait derrière la tisseuse. Happés par le monde des esprits, ils n'ont plus qu'à regarder ébahis la sorcière léviter puis disparaître.

Si dans la scène de la sorcière la forêt a remplacé la lande écossaise, c'est lorsqu'ils atteignent la lisière que les deux cavaliers sont le plus perdus, tournant en rond interminablement dans un espace totalement dépourvu de repères et noyé dans la brume. La ressortie de la forêt fait écho, dans ses «décalages» spatiaux, à la première chevauchée qui y faisait pénétrer Washizu et Miki : le même mouvement indéfiniment répété, mais cette fois circulaire et non plus linéaire, constamment interrompu et reproduit par des changements de plan qui souvent confinent au faux raccord. Mais comment préserver une quelconque cohérence dans ce lieu sans qualités ? Le sol n'y est guère visible que par intermittences, et la brume noie la totalité du décor (existe-t-il seulement ?), à grand-peine percée de loin en loin par les cavaliers, qui nous semblent bien avoir quitté la forêt pour un nulle part sans issue car sans dehors. Seule certitude (tardive), apportée par un plan sans coupures qui confirme ainsi nos pires hantises: les deux hommes tournent en rond de façon plus flagrante et plus circonscrite encore que dans la forêt, lorsqu'ils 
retombaient sur leurs propres traces malgré leur trajectoire apparemment rectiligne. Et puis la brume se dissipe aussi brusquement qu'elle était apparue, mais Washizu et Miki tardent à en tirer la conclusion rassurante : les voilà sortis de la forêt. Celle-ci pourtant était invisible depuis bien longtemps, et lorsque nous percevions les silhouettes des deux cavaliers, ils étaient seuls dans un paysage dénué de tout branchage, de tout feuillage. Nous sommes donc confrontés à un espace fantastique, où la forêt se fait invisible (pour le spectateur) et opaque pour (les personnages) : espace métamorphique, royaume des nuées. Ce lieu indéfinissable (steppe? plateau ?) rappelle l'apologue de Borges sur les deux formes du labyrinthe : l'un repose sur la prolifération des chemins, la saturation des signes; l'autre, plus terrible encore, c'est le désert.

A son retour dans la forêt, en quête explicite de la prophétesse, Washizu vient buter sur un espace prosaïquement inextricable, à la trame aussi concrète qu'impénétrable. Et la caméra filme froidement son errance, son incapacité à trouver l'accès à l'autre monde. C'est en vain qu'il prétend traquer les esprits : ils ne lui apparaîtront que par leur bon vouloir. La sorcière ne cesse de changer d'apparence (de plus en plus explicitement masculine) et d'oripeaux, ct s'amusc à apparaître et à disparaître aux yeux de Washizu, en un jeu de cache-cache spectral qui trace autour de sa proie un cercle évanescent, tissant une toile mobile sans cesse redéfinie mais néanmoins implacable. C'est la toile de l'araignée, qui donne son titre au film comme à la forêt, et dont le châtcau n'est pas le centre mais l'extrémité du fil. C'est aussi bien sûr la toile que tisse la sorcière, dont nous ne percevons que la rotation lancinante du métier : la grande roue cosmique entraîne celle, plus petite, des affaires et des ambitions humaines. Lorsque le contrechamp nous offre le point de vue des csprits, le mouvement évidemment s'inverse pour notre regard, mais sans remettre en question l'irréversibilité de ce processus cyclique, celui que célèbre ct déplore tout à la fois le chœur qui encadre (et encercle) le récit : «Les voies du démon ont de tout temps été vouées à l'échec. Jusqu'au Jugement dernier il en sera toujours ainsi».

Ce caractère mouvant de la forêt, que le sacrilège du régicide a privée d'ancrage, chaos spatial répondant au chaos métaphysique, vient bien sûr directement de Shakespeare. À l'énoncé de la seconde prophétie qui proclame croit-il son invincibilité, Washizu s'exclame comme Macbeth : «Une forêt ne peut bouger». C'est compter naturellement sans l'ironie divine, mais aussi, dans le film, sans l'homologie constante entre forêt et nuée, où l'enracinement n'est qu'un faux-semblant de l'impalpable. Le «Who can impress the forest ?» shakespearien peut alors s'entendre au sens de : Qui peut prétendre fixer pour l'œil ce lieu de l'impossible, matérialiser l'imaginaire, lui donner l'apparence du réel ? La forêt est l'endroit où l'ennemi est censé s'égarer: mais ce sont les protagonistes qui de façon 
répétée s'y perdent, tandis que l'ennemi finit par faire corps avec cette instance surnaturelle, alliée d'un moment contre l'impie possédé par l'hubris. Mais l'illusion d'une forêt qui avance sur la forteresse n'est si parfaite que d'être baignée d'un: nuée complice. Et au dénouement, tandis que la nuée gagne et dévore ses troupes ralliées à l'ennemi, Washizu percé de flèches devient luimême homme-forêt. Non cette fois une menace intangible donc invulnérable, mais au contraire, même s'il se refuse à capituler, une proie entravée. ćcraséc du poids concret de sa mortalité : le bois des flèches, leur sifflement dans l'air, leur impact mat lorsqu'elles pénètrent dans les remparts ou dans la cuirasse donnent soudain à la mort la matérialité palpable d'un réel insistant. À cet instant, terrassé par les spectres, Washizu bascule dans la lourdeur du cadavre.

C'est donc au moment de la défaite, du châtiment d'une ambition criminelle, que les corps retrouvent une épaisseur physique, présence éphémère qui ne parasite qu'un instant la sphère du surnaturel, dont l'cxistence est à la fois plus durable et plus impalpable. Le bruit et la fureur font place au silence, les murailles aux nuées, et la vision se dissout dans un ultime fondu. Le vertige verbalisé par Macbeth, pièce et personnage, affecte ici l'image même. On passe du constat du néant à une image qui, non contente de figurer la «dusty death», est elle-même vouée à l'effacement. Comme si le film tout entier, jusque dans sa texture, n'était que vanité.

Serge CHAUVIN Université de Paris X - Nanterre 